\section{G114(P) THE EFFECTS OF SLEEPING POSITION ON THE VENTILATORY RESPONSE TO HYPOXIA AND HYPERCARBIA}

T Rossor, K Ali, R Trenear, S Hannam, GF Rafferty, A Greenough. Division of Asthma, Allergy and Lung Biology, King's College London, London, UK

\subsection{6/archdischild-2015-308599.113}

Aims The association of a higher rate of sudden infant death syndrome with prone compared to supine sleeping is well documented; the odds ratio for prone versus supine sleeping being 13.9. The mechanisms, however, remain uncertain. Our aims, therefore, were to determine if prone compared to supine sleeping was associated with impaired responses to hypoxia and hypercarbia.

Methods Infants born at 36 weeks post menstrual age or greater without respiratory problems were recruited from the postnatal ward. Physiological measurements were carried out when the infants were in quiet sleep. The hypoxic challenge was delivered via a nasal mask using 15\% oxygen. Respiratory flow was measured using a pneumotachograph connected to the mask. Data were acquired and displayed in real time. Tidal volume was determined by digital integration of the flow signal. Minute volume (MV) was calculated on a $10 \mathrm{~s}$ average. In both positions, baseline ventilation was measured for five minutes whilst the infant was breathing medical air and then the infant's ventilatory response to inhalation of $15 \%$ oxygen was assessed for five minutes. The challenge was stopped if the oxygen saturation dropped to $85 \%$. Infants respond to hypoxia with a biphasic response, first an increase then a decline in ventilation. The maximum MV, the hypoxic decline in MV and the time to the hypoxic decline were calculated. In each position, following a washout period, $4 \%$ carbon dioxide $\left(\mathrm{CO}_{2}\right)$ in air was delivered for five minutes. The magnitude of increase in $\mathrm{MV}$ and the time constant of the increase in MV were calculated.

Results Fifty infants were studied. During exposure to $15 \%$ oxygen, the time to the start of the hypoxic decline was shorter in the prone compared to the supine position (81 s (6-295) versus $155 \mathrm{~s}(2-307)), \mathrm{p}=0.02$. The time constant of the response to $4 \% \mathrm{CO}_{2}$ was longer in the prone compared to the supine position $(68 \mathrm{~s}(5-250)$ versus $36 \mathrm{~s}(3-220)), \mathrm{p}=0.022$.

Conclusion Our results demonstrate a damped response to ventilatory challenges in the prone compared to the supine position.

\section{G115(P) GENETIC AND EPIGENETIC VARIATIONS AND GENE METHYLATION IN INFANTS EXPOSED TO METHADONE IN-UTERO}

${ }^{1} \mathrm{C}$ Gillis, ${ }^{2} \mathrm{P}$ McLaughlin, ${ }^{2} \mathrm{D}$ Osselton, ${ }^{2} \mathrm{~T}$ Hickish, ${ }^{1,3} \mathrm{H}$ Mactier. ${ }^{1}$ Neonatology, Princess Royal Maternity Hospital, NHS GG\&C, Glasgow, UK; '2Department of Archaeology, Anthropology and Forensic Science, Bournemouth University, Bournemouth, UK; ${ }^{3}$ University of Glasgow, University of Glasgow, Glasgow, UK

\subsection{6/archdischild-2015-308599.114}

Aims Maintenance methadone for the treatment of opioid addiction in pregnancy is commonly associated with neonatal abstinence syndrome (NAS). NAS cannot be predicted in individual babies; differences may be explained at least in part by genetic variations. Gene function is also influenced by DNA methylation. We investigated whether single nucleotide polymorphisms (SNPs) in genes involved in methadone metabolism are associated with NAS, as well as methylation of these genes in opioid-dependent mothers and their babies and in controls.
Methods 21 methadone-prescribed opioid-dependent mother/ infant pairs and 32 control mother/infant pairs. All babies were $>36$ weeks' gestation. Controls were selected as either nonsmoking, DEPCAT 1-3 (affluent, $\mathrm{n}=15$ ) or smoking, DEPCAT 4-7 (deprived, $\mathrm{n}=17$ ).

Buccal swabs were obtained for DNA analysis from mother/ infant pairs within 5 days of birth. NAS was defined as symptoms severe enough to require pharmacological treatment.

Results Multiple different SNPs were analysed for 5 opioidrelated genes. Methadone-exposed infants who required treatment for NAS were more likely to carry the wild type (normal) homozygous genotype at CYP2B6 516GT and 785AG compared to infants who did not require treatment.

\begin{tabular}{|c|c|c|c|c|}
\hline & \multicolumn{4}{|c|}{ СYP2B6 } \\
\hline & \multicolumn{2}{|l|}{516} & \multicolumn{2}{|l|}{785} \\
\hline & GG & GT & AA & AG \\
\hline No NAS treatment & 1 & 7 & 2 & 8 \\
\hline NAS treatment & 8 & 2 & 7 & 2 \\
\hline$P$ value & 0.015 & & 0.023 & \\
\hline
\end{tabular}

Infants exposed to methadone in-utero had significantly increased methylation of OPRM1 (receptor), ABCB1 (transporter) and CYP2D6 (metabolising) genes compared to controls ( $\mathrm{p}<0.005)$. Opioid-dependent mothers had increased methylation in only ABCB1 and CYP2D6 genes compared to controls.

Conclusion Infants with the homozygous CYP2B6 genotype are more likely to require treatment for NAS, consistent with the homozygous normal genotype being associated with faster metabolism of methadone.

We have also shown that opioid dependency in pregnancy is associated with significant increases in methylation of at least three opioid genes in the newborn.

Awareness of infant genotype may predict the severity of NAS and has potential to influence management of the neonate.

\section{G116(P) THE HUMAN GUT IS PROBABLY STERILE AT BIRTH}

${ }^{1} \mathrm{~S}$ Khan, ${ }^{1} \mathrm{R}$ Hansen, ${ }^{2} \mathrm{KP}$ Scott, ${ }^{2} \mathrm{JC}$ Martin, ${ }^{3} \mathrm{SH}$ Berry, ${ }^{3} \mathrm{M}$ Stevenson, ${ }^{1} \mathrm{~A}$ Okapapi, ${ }^{3} \mathrm{GL}$ Hold, ${ }^{1} \mathrm{M}$ Munro. ${ }^{1}$ Neonatology, NHS Grampian, Aberdeen, UK; ${ }^{2}$ Rowett Institute of Nutrition and Health, University of Aberdeen, Aberdeen, UK; ${ }^{3}$ Gastrointestinal Research Laboratory, University of Aberdeen, Aberdeen, UK

\subsection{6/archdischild-2015-308599.115}

Aims Considerable effort has been made to categorise the bacterial composition of the human gut and correlate findings with gastrointestinal disease. The infant gut has long been considered sterile at birth followed by rapid colonisation by pioneer microbiota. We examined first-pass meconium from healthy term infants to confirm/refute sterility.

Methods Healthy mothers were approached following vaginal delivery. First-pass meconium within $24 \mathrm{~h}$ of delivery were obtained from healthy, breastfed infants. Antibiotic use was an exclusion criterion- mother within 7 days or infant after birth. Stools were processed in triplicate for fluorescent in-situ hybridisation (FISH) with16S rRNA-targeted probes against all bacteria; Bifidobacterium; Bacteroides-Prevotella; Lactobacillaceael Enterococcaceae; Enterobacteriaceae; Streptococcaceae; Staphylococcaceae and Enterococcaceae. Absolute counts of all bacteria and proportional identification for each bacterial group were calculated. DNA extraction followed by universal bacterial RT-PCR was performed on FISH-positive samples. 